\title{
LIBERALISMO POLÍTICO: MORAL, NO EPISTEMOLÓGICO *
}

\author{
Luciano Venezia \\ Universidad Nacional de Quilmes
}

RESUMEN. El presente ensayo constituye una respuesta crítica a «Liberalismo político, justificación pública y verdad» de M. GARRETA LECLERCQ. GARRETA LECLERCQ sostiene que la teoría de la justificación epistémica tradicional es conceptualmente irreconciliable con el liberalismo político rawlsiano. Asimismo, GARRETA LECLERCQ también mantiene que la concepción de la justificación política de RAWLS sí satisfaría los estándares liberal-políticos pero que sería deficiente por otras razones. Por esta cuestión, GARRETA LECLERCQ concluye que RAWLS no desarrolla una justificación adecuada del núcleo conceptual del liberalismo político. Por mi parte, considero que en Political Liberalism, RAWLS da cuenta de la neutralidad liberal sobre la base de razones morales antes que epistemológicas. Por esta razón, los argumentos esgrimidos por GARRETA LECLERCQ no ofrecen elementos de juicio suficientes para afirmar que RawLS introduce el núcleo de ideas característico del liberalismo político de modo inadecuado.

Palabras clave: liberalismo político, justificación pública, razonabilidad, verdad.

ABSTRACT. This essay is a critical response to M. GARRETA LECLERCQ's «Liberalismo político, justificación pública y verdad». GARRETA LECLERCQ argues that the traditional theory of epistemic justification is conceptually irreconcilable with Rawlsian political liberalism. In addition, GARRETA LECLERCQ holds that even though RAWLs's own conception of political justification satisfies political liberalism's standards, it would be objectionable on other grounds. On the basis of such considerations, GARRETA LECLERCQ concludes that RAWLS does not develop an adequate justification of political liberalism's conceptual core. I disagree. I argue that in Political Liberalism, RAWLS grounds liberal neutrality on the basis of moral rather than epistemological reasons. I conclude that GARRETA LECLERCQ's arguments fail to provide adequate support for claiming that RAWLS grounds the conceptual core of political liberalism in a misguided way.

Keywords: political liberalism, public justification, reasonableness, truth.

* Fecha de recepción: 12 de mayo de 2010. Fecha de aceptación: 20 de junio de 2010.

Una versión anterior de este trabajo fue presentada el 27 de marzo de 2010 en las III Jornadas CIFSADAF, «Autoconocimiento, democracia deliberativa y justicia global», en la Sociedad Argentina de Análisis Filosófico en Buenos Aires, Argentina. Agradezco la invitación de Eduardo Rivera López y la buena disposición de Mariano Garreta Leclercq para discutir sus ideas conmigo. Asimismo, agradezco los comentarios de Pablo Stafforini a una versión anterior del ensayo. Por último, agradezco las sugerencias de el/la evaluador(a) anónimo/a de Doxa, Cuadernos de Filosofía del Derecho. 


\section{INTRODUCCIÓN}

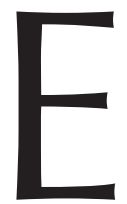

1 presente ensayo tiene por objetivo examinar críticamente algunas de las ideas desarrolladas por M. GARRETA LECLERCQ en «Liberalismo político, justificación pública y verdad». La argumentación desarrollada por GARRETA LECLERCQ en este texto está estructurada en dos partes. En la primera parte, GARRETA LECLERCQ argumenta que la teoría de la justificación epistémica tradicional es conceptualmente irreconciliable con el liberalismo político rawlsiano -en los hechos, esta teoría conllevaría la adopción de una posición escéptica acerca del valor de las creencias filosóficas, religiosas o morales que abrazan los ciudadanos razonables en el ámbito no-público o privado-. Asimismo, GARRETA LECLERCQ mantiene que la concepción de la justificación política de RAWLS sí cumpliría con el estándar anti-escéptico liberal-político, pero que sería deficiente por otras razones -en particular, por no ser una genuina teoría de la justificación capaz de competir con la concepción epistémica tradicional, debido a que (entre otras cosas) el concepto de verdad no jugaría un papel relevante en esta concepción-. Sobre la base de esta argumentación, GARRETA LECLERCQ concluye que RAWLS no desarrolla una concepción de la justificación epistémica verdaderamente atendible del núcleo conceptual del liberalismo político - la neutralidad de justificación-, sino que introduce una teoría dudosa de forma «axiomática». En la segunda parte del ensayo, GARRETA LECLERCQ propone una forma novedosa para subsanar el problema detectado. Allí GARRETA LECLERCQ desarrolla una concepción de la justificación pública que estaría en condiciones de pasar el test anti-escéptico característico del liberalismo político, al mismo tiempo que no sería presa de las dificultades detectadas en la argumentación de RAWLS.

En este trabajo focalizo mi argumentación en la parte destructiva de la argumentación desarrollada por GARRETA LECLERCQ en su artículo. En particular, en este ensayo muestro que RAWLS da cuenta de la neutralidad liberal sobre la base de razones morales antes que epistemológicas. Por ello, los argumentos críticos de GARRETA LECLERCQ no ofrecen elementos de juicio suficientes para afirmar que RAWLS justifica el núcleo de ideas característico del liberalismo político sobre la base de la adopción axiomática de una concepción deficiente de la justificación pública. Con todo, los diferentes puntos que analizo en este trabajo no alcanzan a la teoría positiva desarrollada por GARRETA LECLERCQ. Consiguientemente, mi argumentación no es totalmente incompatible con su proyecto teórico. Aun cuando aceptara los argumentos que desarrollo a continuación, GARRETA LECLERCQ podría de todos modos considerar que existen razones adicionales para introducir su propia concepción de la justificación pública, al margen de que fracase o no la justificación de RAWLS de la neutralidad liberal.

\section{MERO PLURALISMO VS. PLURALISMO RAZONABLE}

Una forma de plantear el núcleo argumentativo desarrollado por GARRETA LECLERCQ en su artículo es la siguiente. Contrariamente a lo que dice hacer, en los hechos RAWLS asume una posición escéptica acerca del valor de las doctrinas comprehensivas filosóficas, religiosas o morales que los ciudadanos razonables sostienen en el ámbito 
privado o no-público. La argumentación de RAWLS introduce una distinción conceptual entre «creencias comprehensivas como tales» y «creencias comprehensivas verdaderas» ${ }^{1}$. A la luz de esta distinción, RAWLS sostiene que las doctrinas comprehensivas no constituyen una base adecuada para sustentar una concepción de la justicia distributiva. La argumentación de RAWLS involucra la idea de que las doctrinas comprehensivas no son verdaderas o correctas, lo que conlleva la introducción de un juicio escéptico acerca de su valor epistémico - equivale a decir que las doctrinas comprehensivas no están adecuadamente justificadas y, quizá, a que son lisa y llanamente falsas-.

GARRETA LECLERCQ desarrolla un ejemplo para mostrar la introducción de este juicio escéptico de la argumentación rawlsiana. El ejemplo consiste en imaginar a un científico explicándole a un lego una teoría compleja. Según la forma en que GARRETA LECLERCQ plantea las cosas, el lego no comprende la explicación y, dado que ella choca con el sentido común, él considera que la teoría en cuestión es falsa ${ }^{2}$. Ahora bien, GARRETA LECLERCQ señala que, intuitivamente, en este caso no diríamos que el científico no dispone de una justificación apropiada de su teoría o que falló en justificar esa teoría frente a su interlocutor. Seguramente diríamos que el científico no logró persuadir al lego. Sin embargo, no diríamos que falló en ofrecer una explicación de la teoría ${ }^{3}$.

GARRETA LECLERCQ señala que este ejemplo tiene un estatus fundamentalmente análogo al caso de una persona razonable que intentara defender su propio punto de vista comprehensivo frente a otros ciudadanos razonables en el ámbito público. Ahora bien, aceptar la analogía conlleva admitir del carácter escéptico de la posición de RAWLS. En efecto, mientras que nuestra evaluación de la situación sería sustancialmente similar a la evaluación del caso del científico (la persona razonable habría justificado su posición aunque tal vez no hubiera convencido a su interlocutor), RAWLS, en tanto abraza la tesis de la neutralidad de justificación, evaluaría la situación de otro modo. Él asumiría que la persona no está realmente justificada en sostener ese punto de vista comprehensivo. Así, en los hechos, RAWLS estaría adoptando un punto de vista escéptico acerca del valor de las concepciones comprehensivas de los ciudadanos razonables ${ }^{4}$.

A mi juicio, esta conclusión es apresurada. Los dos casos no son realmente similares en los aspectos o características relevantes. En realidad, los dos casos son lo suficientemente heterogéneos entre sí como para invalidar la analogía planteada por GARRETA LECLERCQ. El elemento de juicio clave es el siguiente. Mientras que en el caso del científico podemos considerar que nos encontramos en una situación en la que, en principio, es posible ofrecer una justificación de la teoría en cuestión, tal cosa no ocurre en el caso de la persona razonable que intenta dar cuenta de su punto de vista comprehensivo en el ámbito público frente a otros ciudadanos razonables que no comparten ese punto de vista. En el primer caso no existen obstáculos insalvables para que el lego comprenda la explicación y adquiera los conocimientos provistos por el científico. Con tiempo y esfuerzo, el lego podría comprender la explicación. Sin embargo, ello no podría tener lugar en el caso de la defensa de un punto de vista comprehensivo

GARRETA LeCLERCQ, 2011: 5-6. Todas las citas corresponden al manuscrito escrito en MS Word,

Vid. GARRETA LECLERCQ, 2011: 8 .

Vid. GARRETA LECLERCQ, 2011: 8-9.

Vid. GARRETA LeCLercQ, 2011: 9. 
en el ámbito público. Es precisamente esta la diferencia entre el mero pluralismo que puede observarse en los desacuerdos que se dan en el ámbito de las ciencias fácticas ${ }^{5}$ y el pluralismo razonable que se presenta en el plano de las doctrinas comprehensivas en las sociedades democráticas modernas.

En el caso del científico, es posible en principio ofrecer una justificación de la teoría en cuestión. Sin embargo, el hecho del pluralismo razonable conlleva la idea de que existen obstáculos insalvables para la justificación de un punto de vista comprehensivo en el ámbito público. Aunque existan razones perfectamente legítimas para quienes afirman una doctrina comprehensiva particular, ello no significa que esas mismas razones sean suficientes o siquiera relevantes para que otros ciudadanos razonables acepten ese punto de vista comprehensivo. Asumamos (como por otro lado hace el propio RAWLS ${ }^{6}$ que las principales religiones monoteístas adoptadas por muchos ciudadanos de las democracias occidentales son razonables. Consiguientemente, deberíamos considerar razonable la creencia de que realizar un aborto es un asesinato porque Dios da alma al feto en el momento de la concepción ${ }^{7}$. Por tanto, también deberíamos aceptar que muchos creyentes religiosos tienen buenas razones para creer tal cosa (por ejemplo, porque esa creencia está articulada y fundamentada por elementos de juicio aceptables para las personas educadas y formadas en esa tradición, como dogmas religiosos, documentos oficiales de la Iglesia, declaraciones de las máximas autoridades eclesiásticas, etc.). Con todo, ello no significa que personas razonables que no compartan ese punto de vista comprehensivo (v.g., agnósticos o ateos) deban aceptar las razones que tienen los creyentes religiosos para creer tal cosa. Estas personas quizá no acepten la idea misma de Dios, o tal vez tengan serias dudas respecto de su existencia, por lo que difícilmente aceptarían las razones de los creyentes religiosos para rechazar el aborto.

Hasta aquí únicamente enfaticé las diferencias entre los dos casos. Pero hay un elemento más a tener en consideración. Intuitivamente, parece claro que un creyente religioso difícilmente aceptaría no haber ofrecido razones para creer en la verdad o corrección de la tesis en cuestión. En realidad, dadas sus creencias religiosas, su educación, etc., el creyente seguramente consideraría que ha ofrecido genuinas razones para justificar su rechazo del aborto. Sin embargo, quienes no comparten ese punto de vista no considerarían que no han sido persuadidos por las razones traídas a colación; en cambio, esas personas seguramente considerarían que no se han presentado razones en absoluto y, por tanto, que el creyente no ha logrado justificar su punto de vista comprehensivo frente a ellos. Sin embargo, ello no es lo que ocurre en el ejemplo del científico. De allí que la analogía entre los dos casos se rompa.

Ahora bien, aceptar que una persona razonable no lograría justificar su propio punto de vista comprehensivo frente a otros ciudadanos razonables que no comparten ese conjunto de ideas no conlleva la idea de que aquellos que no comparten ese punto de vista estén equivocados o no tengan una justificación adecuada de sus propias concepciones comprehensivas. De hecho, recién asumimos que esas personas eran razonables, i.e., que tenían buenas razones para justificar sus propios puntos de vista

5 Vid. RAWLS, 1996: 55. RAWLS señala que en el caso de las ciencias naturales es posible esperar lograr llegar a un acuerdo, al menos en el largo plazo.

6 Vid. RAWLS, 1996: 59.

7 GARRETA LECLERCQ, 2011: 11, introduce este ejemplo. 
comprehensivos, del mismo modo que también asumimos que el creyente religioso también era razonable en el sentido señalado. Por tanto, si estas personas son verdaderamente razonables, ellas comprenderían que lo que cuenta como una buena razón para ellos mismos, o para quienes comparten su mismo punto de vista comprehensivo, no tendría necesariamente ese rol en el caso de aquellos que adhieren a concepciones comprehensivas razonables diferentes.

Por cierto, estimo que GARRETA LECLERCQ no debe estar satisfecho con mi evaluación del ejemplo. Él señala que RAWLS adopta una actitud escéptica respecto de las creencias de los ciudadanos razonables en los hechos, por lo que seguramente podría decir que asumir que un creyente religioso no podría justificar su punto de vista comprehensivo frente a los interlocutores que no compartiesen esa perspectiva comprehensiva conlleva la idea de que esas convicciones tendrían un estatus meramente subjetivo y no estarían adecuadamente justificadas. Sin embargo, creo que no tenemos que aceptar esta idea. La forma en que una persona razonable evalúa las cosas no tiene por qué verse afectada por la existencia del hecho del pluralismo razonable. En particular, lo que para una persona razonable cuenta como una buena razón no tiene por qué verse conmovido por el hecho de que otras personas igualmente razonables no consideren o evalúen las cosas del mismo modo. En cambio, la idea fundamental es que si esta persona es razonable en el sentido indicado, entonces ella tendría el deseo de encontrar principios que todos los ciudadanos razonables pudieran aceptar y no principios que serían aceptables únicamente para aquellas personas que compartiesen su propia doctrina comprehensiva. Dado el hecho del pluralismo razonable, la forma en que él articule y justifique la comprehensiva religiosa que abraza en el plano nopúblico o privado no tiene por qué ser satisfactoria o siquiera relevante para aquellos que tienen un punto de vista comprehensivo razonable diferente. El hecho de que sus creencias normativas estén articuladas con dogmas religiosos, documentos oficiales de la Iglesia, declaraciones de las máximas autoridades eclesiásticas, etc., bien puede ser suficiente para que considere que su propio punto de vista está adecuadamente justificado. Sin embargo, si es razonable, esa persona también comprendería que las personas razonables que adoptan un punto de vista diferente en materia religiosa no considerarían que esos elementos de juicio son relevantes y mucho menos que son suficientes para justificar una concepción de la justicia distributiva. Ver las cosas de una forma diferente conlleva negar la existencia misma del hecho del pluralismo razonable y, por ello, constituye un modo de actuar típicamente irrazonable.

De este modo, acepto que si fuera el caso de que RAWLS asumiera en los hechos una posición escéptica respecto del valor de las creencias asociadas a las doctrinas comprehensivas que sostienen los ciudadanos razonables en el ámbito no-público o privado, entonces su posición sería pasible del tipo de crítica que realiza GARRETA LECLERCQ. En este caso, la argumentación de RAWLS no cumpliría adecuadamente con los estándares anti-escépticos necesarios para justificar adecuadamente la neutralidad liberal. Pero RAWLS no comete este pecado moral, de forma tal que su argumentación no es pasible de ese tipo de crítica. En el contexto del marco teórico desarrollado por RAWLS, la neutralidad liberal está justificada en la existencia del deseo de las personas razonables de encontrar principios que todos los ciudadanos razonables puedan aceptar antes que en principios que sean aceptables únicamente para aquellos que afirman una doctrina comprehensiva particular. 


\section{LIBERALISMO POLÍTICO: MORAL, NO EPISTEMOLÓGICO}

Según veo las cosas, en Political Liberalism, RAWLS no adopta una posición epistemológica para dar cuenta de la neutralidad de justificación característica del liberalismo político. Más bien, sus ideas en este sentido consisten en evitar tomar partido acerca de la justificación de las doctrinas comprehensivas que los ciudadanos razonables afirman en el ámbito no-público o privado, i.e., evitar evaluarlas a la luz de una teoría de la justificación epistémica. En realidad, la estrategia argumentativa de RAWLS no sólo no involucra cuestionamiento alguno respecto de la justificación de esas creencias, sino que su argumentación involucra la idea de que realizar ese tipo de evaluación constituye un punto de partida errado. En sociedades marcadas por el hecho del pluralismo razonable, todos los ciudadanos razonables podrían aducir que sus concepciones normativas están realmente justificadas. Este fenómeno motiva el planteamiento de RAWLS de que ciudadanos razonables considerarían que el basamento de una concepción de la justicia distributiva no puede estar constituido por elementos de juicio aceptables únicamente a la luz de elementos de juicio internos a las doctrinas comprehensivas, sino que debe estar articulado sobre la base de razones aceptables para todos los ciudadanos razonables.

De este modo, la distinción señalada por GARRETA LECLERCQ entre «entre creencias comprehensivas que pueden ser afirmadas, en forma justificada, como verdaderas, y creencias comprehensivas que tienen un estatus puramente subjetivo» ${ }^{8}$ es en verdad ajena al planteo rawlsiano. Por supuesto, como señala GARRETA LECLERCQ, RAWLS realiza una distinción entre «creencias comprehensivas como tales» y «creencias comprehensivas verdaderas» en el marco de su argumentación a favor de la neutralidad liberal. Sin embargo, ello no significa que RAWLS considere que las creencias comprehensivas no sean verdaderas o no estén apropiadamente justificadas. En el contexto de una sociedad marcada por el hecho del pluralismo razonable debemos abandonar el espacio de lo verdadero o correcto e introducir en su lugar el de lo razonable no tanto debido al hecho de las doctrinas comprehensivas no sean verdaderas o no estén suficientemente justificadas. Debemos abandonar dicho espacio porque el tipo de razones que podemos dar para justificar esas creencias frente a los demás no sería adecuado. A lo sumo, ellas serían razones aceptables únicamente para aquellos que adopten el mismo punto de vista comprehensivo, pero no serían aceptables por quienes sostienen doctrinas comprehensivas razonables diferentes. La aceptación del hecho del pluralismo razonable en las sociedades democráticas contemporáneas tiene serias consecuencias respecto de la forma en que podemos justificar de una concepción de la justicia distributiva. Pero ello no conlleva introducir de forma subrepticia un juicio escéptico acerca del valor de las distintas doctrinas comprehensivas que afirman los ciudadanos razonables. Así, la tesis rawlsiana de que las doctrinas comprehensivas son razonables y no verdaderas no conlleva afirmar que tienen un estatus meramente subjetivo y no pueden ser adecuadamente justificadas y mucho menos que son falsas o no están justificadas en absoluto.

\footnotetext{
8 GARRETA LECLERCQ, 2011: 18.
} 
La caracterización de RAWLS del hecho del pluralismo razonable está asociada a la consideración del papel que juegan las «cargas del juicio» ${ }^{9}$ en el ejercicio libre de la razón humana al momento de determinar el contenido del bien en el marco de una tradición doctrinal comprehensiva de carácter filosófico, religioso o moral. GARRETA LECLERCQ señala que la incorporación de estos elementos en la argumentación de RAWLS conllevaría la introducción una actitud escéptica respecto del valor de las creencias comprehensivas. Ellas «conducirían a reconocer que la afirmación de que nuestras creencias comprehensivas son verdaderas es una pretensión que nadie está en condiciones de justificar, en forma general, frente al resto de sus conciudadanos en el marco del debate político» ${ }^{10}$. A mi juicio, las cargas del juicio tienen un papel diferente al señalado por GARRETA LECLERCQ en la argumentación de RAWLS a favor de la neutralidad liberal.

Las cargas del juicio no involucran juicio alguno respecto de la actitud que los ciudadanos razonables deberían tomar respecto de las diferentes doctrinas comprehensivas que abrazan. Las cargas del juicio conforman un listado de las circunstancias que hacen que llegar a un acuerdo racional acerca del contenido del bien humano sea difícil o lisa y llanamente imposible ${ }^{11}$. Consiguientemente, las cargas del juicio explican pero, al mismo tiempo, también justifican la pluralidad existente en las sociedades democráticas modernas. En otras palabras, las cargas del juicio no sólo permiten dar cuenta de la pluralidad existente sino que, además, permiten calificar al hecho del pluralismo como razonable. Ahora bien, RAWLS destaca que uno de los rasgos fundamentales de las personas razonables consiste precisamente en aceptar la existencia del hecho del pluralismo razonable ${ }^{12}$. En este sentido, el reconocimiento de la existencia del hecho del pluralismo razonable introduce un límite respecto de aquello que las personas razonables considerarían que puede ser tomado como punto de partida para justificar una concepción de la justicia distributiva. En particular, involucra la idea de que la apelación a la (supuesta) verdad o corrección de una doctrina comprehensiva particular no constituye una razón válida para justificar una concepción de la justicia social y económica ${ }^{13}$. Este modo de proceder desconocería la razonabilidad de las doctrinas comprehensivas diferentes que abrazan los demás ciudadanos razonables. Sin embargo, las personas razonables aceptan la existencia del hecho del pluralismo razonable y, con ello, la existencia de buenas razones para justificar la adopción de diferentes puntos de vista comprehensivos. Por ello, ellas aceptarían que la apelación a elementos de juicio internos a una doctrina comprehensiva particular constituye un punto de partida inadecuado.

De este modo, el reconocimiento de la existencia del hecho del pluralismo razonable involucra una valoración respecto del papel que deben tener las doctrinas comprehensivas en la justificación de una concepción de la justicia distributiva. En el contexto de una sociedad marcada por el hecho del pluralismo razonable, la justificación de una concepción de la justicia distributiva tiene que ser «política» antes que

\footnotetext{
9 Vid. Rawls, 1996, 56. Vid. también Garreta LeClerCQ, 2011: 3-4.

10 GarReta LeCLERCQ, 2011: 3.

11 Vid. RAWLS, 1996: 63.

12 Vid. RAWLS, 1996: 54, 58-59.

13 Vid. RaWls, 1996: 61.
} 
«comprehensiva» porque, eventualmente, todos los ciudadanos razonables podrían aducir la verdad o corrección de su propio punto de vista comprehensivo. Sin embargo, en tanto las personas razonables, ellas se caracterizan precisamente por reconocer la existencia de diferentes doctrinas comprehensivas razonables antes que por considerar solamente la razonabilidad de la propia concepción comprehensiva. Por tanto, ellas considerarían que se debe abandonar el espacio de la verdad o corrección de las diferentes convicciones normativas y asumir el de la razonabilidad. Esta forma de proceder permite asumir la razonabilidad de la propia doctrina comprehensiva al mismo tiempo que aceptar la razonabilidad de las concepciones comprehensivas que abrazan los demás ciudadanos razonables. En cambio, asumir la verdad o corrección de la propia doctrina comprehensiva lógicamente involucra asumir la falsedad o incorrección de los puntos de vista diferentes, cuestionando así su razonabilidad.

Otra forma de plantear esta cuestión podría ser la siguiente. Si nos limitáramos a considerar el valor de verdad de las diferentes doctrinas comprehensivas, seguramente deberíamos consideraríamos a muchas de ellas — quizá a todas ellas- falsas o incorrectas ${ }^{14}$. Ello volvería a estas concepciones normativas indignas de ser tomadas en consideración al momento de justificar principios de justicia destinados a regular la estructura básica de la sociedad. Pero lo haría al costo de introducir un punto de vista escéptico, volviendo con ello al liberalismo político internamente incoherente. Ahora bien, además, esta actitud involucraría desconocer la existencia misma del hecho del pluralismo razonable, i.e., desconocería el hecho de que la pluralidad existente en las democracias modernas es el resultado del ejercicio de la racionalidad humana en sus diferentes aspectos teóricos y prácticos. Por ello, la aceptación de la existencia misma del hecho del pluralismo razonable conlleva la aceptación de la idea de que la adopción de un punto de vista comprehensivo constituye un paso injustificado respecto del tipo de consideraciones que los ciudadanos podrían aducir para justificar una concepción de la justicia distributiva.

Con todo, asumir la razonabilidad de la propia doctrina comprehensiva, así como la razonabilidad de las doctrinas comprehensivas diferentes a la propia, no involucra dejar de visualizar a las propias ideas como verdaderas o correctas. La argumentación de RAWLS no involucra desconocer la forma en que los ciudadanos razonables visualizan sus concepciones comprehensivas, i.e., como la verdad moral, filosófica o religiosa, según sea el caso. El punto clave de la argumentación rawlsiana consiste precisamente en señalar que, en el contexto de una sociedad marcada por el hecho del pluralismo razonable, todos los ciudadanos razonables pueden realizar el mismo tipo de juicio respecto de sus propias concepciones comprehensivas (i.e., sostener que son verdaderas o correctas) sobre la base de razones atendibles, aun cuando esas razones no sean válidas para aquellos que adoptan puntos de vista comprehensivos diferentes. Asimismo, la idea de que ciudadanos razonables reconocen la razonabilidad de sus propias convicciones normativas, así como la razonabilidad de aquellas que son diferentes a las propias, no involucra la idea de que ellos no consideren a sus propios puntos de vista comprehensivos como verdaderos o correctos, al mismo tiempo que consideren a los puntos de vista diferentes como falsos o incorrectos. Una persona razonable que abraza una doctrina comprehensiva particular considera que

14 Vid. RaWls, 1996: 58, 60. 
está en lo cierto y que los que adoptan un punto de vista diferente se equivocan. Pero éste no es el punto fundamental, puesto que si esta persona es razonable, también consideraría que esas otras personas tienen buenas razones para afirmar el punto de vista comprehensivo que sostienen, i.e., consideraría que no son irrazonables aun cuando afirmen una concepción normativa falsa o incorrecta según su forma de ver las cosas.

De este modo, la argumentación rawlsiana no introduce juicio alguno acerca del valor epistémico de las creencias comprehensivas que afirman los ciudadanos razonables. De hecho, RAWLS introduce un estándar deliberadamente vago y poco exigente para dar cuenta de la razonabilidad de las diferentes doctrinas comprehensivas ${ }^{15}$. Por tanto, la idea de que los propios agentes que abrazan una doctrina comprehensiva consideren a ese punto de vista como la verdad moral, filosófica o religiosa es perfectamente compatible con la teoría de RAwLS. Asimismo, ellos también pueden considerar que los que no adoptan ese punto de vista están lisa y llanamente en el error (por cierto, las personas razonables también pueden tomar una actitud diferente respecto del valor de verdad de las convicciones comprehensivas propias y/o diferentes). Esto no es realmente importante. En cambio, el punto clave consiste en destacar la razonabilidad (antes que la verdad o falsedad) de las diferentes doctrinas comprehensivas, así como el hecho de que las personas razonables aceptan la razonabilidad de las diferentes doctrinas comprehensivas aun cuando estén convencidas de que esos puntos de vista sean errados. De este modo, el elemento crucial que introduce el argumento de RAWLS no consiste en cuestionar el valor de verdad o el grado de justificación de las concepciones comprehensivas de los ciudadanos razonables, sino en destacar que el reconocimiento del hecho del pluralismo razonable involucra dejar de lado este tipo de consideraciones, para en cambio asumir un punto de vista neutral al momento de justificar una concepción de la justicia distributiva.

De esta forma, la justificación rawlsiana de la neutralidad liberal no descansa sobre la base de consideraciones epistemológicas, especialmente sobre la base de una distinción «entre creencias comprehensivas que pueden ser afirmadas, en forma justificada, como verdaderas, y creencias comprehensivas que tienen un estatus puramente subjetivo». La argumentación de RAWLS no involucra juicio alguno acerca del valor de verdad o del grado de justificación de las convicciones filosóficas, religiosas o morales que afirman los ciudadanos razonables en el ámbito no-público o privado. En cambio, RAWLS asume la razonabilidad de las diferentes doctrinas comprehensivas, i.e., asume la existencia del hecho del pluralismo razonable. Por ello, el basamento rawlsiano de la neutralidad liberal es moral antes que epistemológico, en tanto se funda en el deseo de los ciudadanos razonables de encontrar principios que los demás ciudadanos razonables puedan aceptar. Dado el hecho del pluralismo razonable, ese deseo conlleva el reconocimiento de que buscar imponer el propio punto de vista normativo asociado a la doctrina comprehensiva que uno afirma en el ámbito no-público o privado es fundamentalmente irrazonable. Esta actitud involucra el desconocimiento de la razonabilidad de los puntos de vista comprehensivos diferentes del propio y, con ello, desconoce la existencia misma del hecho el pluralismo razonable. 


\section{BIBLIOGRAFÍA}

GARRETA LECLERCQ, M., 2011: «Liberalismo político, justificación pública y verdad», en Doxa, Cuadernos de Filosofía del Derecho, núm. 33.

RaWLs, J., 1996: Political Liberalism; with a New Introduction and the «Reply to Habermas», edición en rústica (New York, Columbia University Press). 